\title{
A Catalogue of carbon stars in the LMC*
}

\author{
E. Kontizas ${ }^{1}$, A. Dapergolas ${ }^{1}$, D. H. Morgan ${ }^{2}$, and M. Kontizas ${ }^{3}$ \\ 1 Institute of Astronomy and Astrophysics, National Observatory of Athens, PO Box 20048, 11810 Athens, Greece \\ 2 Institute for Astronomy, University of Edinburgh, Blackford Hill, Edinburgh EH9 3HJ, UK \\ 3 Section of Astrophysics Astronomy and Mechanics, Department of Physics, University of Athens, 15783 Athens, \\ Greece
}

Received 2 August 2000 / Accepted 23 January 2001

\begin{abstract}
A catalogue of 7760 carbon stars in the Large Magellenic Cloud is presented. The stars were identified during a systematic survey of objective-prism plates taken with the UK $1.2 \mathrm{~m}$ Schmidt Telescope. The catalogue is compared with other lists of carbon stars and the distribution of the carbon stars is discussed.
\end{abstract}

Key words. galaxies: Magellanic Clouds - stars: carbon

\section{Introduction}

The spatial distributions of a galaxy's component stellar populations are very good tracers of the galaxy's star formation and evolution. Carbon stars characterize wellevolved stars and represent a wide range of intermediate stellar masses. With the Magellanic Clouds having a high proportion of intermediate-mass stars, it is very important to study the space distributions of their carbon stars.

Complete spatial coverage of the carbon star population in the Small Magellanic Cloud (SMC) has been achieved through the spectral surveys by Rebeirot et al. (1993) and Morgan \& Hatzidimitriou (1995); but coverage of the carbon stars in the Large Magellanic Cloud (LMC) is still very limited: Blanco et al. (1980) and Blanco \& McCarthy $(1983,1990)$ have published a survey of M-type and carbon stars seen on grism plates of 52 small circular (0.12 sq deg) selected areas, and Kunkel et al. (1997) have presented lists of carbon stars found in the outer regions of both the SMC and the LMC from the photometric selection of very red stars.

This paper describes the results of an extensive programme of identifying carbon stars throughout the entire LMC using objective-prism plates taken with the UK $1.2 \mathrm{~m}$ Schmidt Telescope (UKST) at Siding Spring Observatory, Australia. The carbon stars found are presented in a catalogue in electronic form which gives the po-

\footnotetext{
Send offprint requests to: E. Kontizas,

e-mail: ekonti@astro.noa.gr

* Tables 3-5 are only available in electronic form at the CDS via anonymous ftp to csarc.u-strasbf.fr (130.79.128.5) or via

http://cdsweb.u-strasbg.fr/cgi-bin/qcat?J/A+A/369/932
}

sitions and photographic photometry of 7760 carbon stars. Sections 2-4 describe the observational data and survey techniques, the photometric calibration of the data and the Catalogue itself. There is a discussion of the completeness of the Catalogue in Sect. 5, including comparisons with published lists of carbon stars. Finally, the overall distribution of the carbon stars is described in Sect. 6 .

\section{Observations and measurements}

The observational source material consists of twelve deep objective-prism plates taken with the UKST and covers an area of $\sim 300 \mathrm{sq}$ deg. The plates are mostly those used for the UKST Planetary Nebula Survey (Morgan 1994); details are given in Table 1.

All the plates are sky-limited exposures on Kodak IIIa-J emulsion taken through a Schott GG455 filter and an objective prism of dispersion $800 \AA \mathrm{mm}^{-1}$ near $\mathrm{H} \gamma$. The spectra are dispersed north-south and unwidened. See Savage et al. (1985a, 1985b) for further details of the prisms and illustrations of carbon stars as they appear on UKST plates. The plates cover almost the whole area of LMC and more than $40 \%$ of this area is common to two or more plates.

The plates were searched by systematic, visual scanning using a microscope at $\times 16$ magnification. The carbon stars were identified on the basis of the prominent Swan $C_{2}$ bands at $4737 \AA$ and $5165 \AA$. Many carbon stars were easily identified on these plates and could be seen down to $b_{\mathrm{J}} \sim 19.5$. This survey is similar to the one carried out using UKST plates of the outer regions of the SMC by Morgan \& Hatzidimitriou (1995), so the vast majority of the candidates should be carbon stars, as 
Table 1. Plates used in the carbon star survey

\begin{tabular}{lcccrc}
\hline Plate & $F$ & \multicolumn{2}{c}{ Centre } & Exp & Date \\
& & RA & Dec & & \\
\hline YJ 13472P & 32 & $4^{\mathrm{h}} 24^{\mathrm{m}}$ & $-75^{\circ} 00^{\prime}$ & 90 & 30 Nov. 1989 \\
YJ 11510P & 33 & $5^{\mathrm{h}} 30^{\mathrm{m}}$ & $-75^{\circ} 00^{\prime}$ & 120 & 25 Nov. 1986 \\
YJ 15250P & 34 & $6^{\mathrm{h}} 36^{\mathrm{m}}$ & $-75^{\circ} 00^{\prime}$ & 110 & 26 Nov. 1992 \\
YJ 10677P & 55 & $4^{\mathrm{h}} 20^{\mathrm{m}}$ & $-70^{\circ} 00^{\prime}$ & 90 & 13 Jan. 1986 \\
YJ 12949P & 56 & $5^{\mathrm{h}} 12^{\mathrm{m}}$ & $-70^{\circ} 00^{\prime}$ & 80 & 2 Feb. 1989 \\
YJ 10678P & 57 & $6^{\mathrm{h}} 04^{\mathrm{m}}$ & $-70^{\circ} 00^{\prime}$ & 62 & 13 Jan. 1986 \\
YJ 13469P & 84 & $4^{\mathrm{h}} 24^{\mathrm{m}}$ & $-65^{\circ} 00^{\prime}$ & 90 & 29 Nov. 1989 \\
YJ 14039P & $\mathrm{N} 3$ & $5^{\mathrm{h}} 08^{\mathrm{m}}$ & $-62^{\circ} 45^{\prime}$ & 120 & 11 Dec. 1990 \\
YJ 11656P & 86 & $5^{\mathrm{h}} 52^{\mathrm{m}}$ & $-65^{\circ} 00^{\prime}$ & 135 & 25 Feb. 1987 \\
YJ 12873P & 87 & $6^{\mathrm{h}} 36^{\mathrm{m}}$ & $-65^{\circ} 00^{\prime}$ & 95 & 5 Dec. 1988 \\
YJ 8312P & $(\mathrm{S})$ & $5^{\mathrm{h}} 30^{\mathrm{m}}$ & $-72^{\circ} 12^{\prime}$ & 90 & 17 Dec. 1982 \\
YJ 9007P & $(\mathrm{N})$ & $5^{\mathrm{h}} 20^{\mathrm{m}}$ & $-66^{\circ} 48^{\prime}$ & 90 & 1 Feb. 1984 \\
\hline
\end{tabular}

Exp is the exposure in minutes.

$F$ is usually the ESO/SERC Field Number.

$(\mathrm{N})$ and $(\mathrm{S})$ are commonly used, non-standard centres.

The coordinates are B1950.

was found in subsequent spectroscopy of the SMC stars (Hatzidimitriou et al. 1997). At the plate scale of the UKST $\left(67.14 \operatorname{arcsec} \mathrm{mm}^{-1}\right)$, these spectra are $\sim 0.5 \mathrm{~mm}$ long, so crowding is not a problem over much of the area covered. However, it is important in the inner regions which, though small in area, do contain a relatively high proportion of the carbon stars. The topic of completeness will be discussed later.

The positions of the stars were obtained from plates taken for the SR-component of the SERC-I/SR Survey of the Milky Way and the Magellanic Clouds. (See Morgan et al. 1992 for details of this and other surveys used). The measurements were made by the SuperCOSMOS fast measuring machine at the Royal Observatory Edinburgh, details of which can be found on the SuperCOSMOS Web Site - http://www.roe.ac.uk/cosmos/scosmos.html. Identification of a carbon star in these datasets was achieved by extracting and plotting as a finding chart all the SuperCOSMOS images in 1.5 arcmin boxes around an approximate position which had been obtained in the manner described by Dapergolas et al. (1996) to an accuracy of $\sim 30$ arcsec. The carbon star was then identified by comparing this chart with the objective-prism plate, with the help of an overlaid film copy of the SR Survey. The SuperCOSMOS coordinates were then stored. The identification process was straightforward in all but the most crowded areas. Measurements of stars located on more than one field were almost always taken from the field in which they were more central.

Both components of the I/SR Survey were measured to provide photometry of the carbon stars in two wavebands. The image parameters provided by SuperCOSMOS include an uncalibrated magnitude, the image shape, a measurement of the local plate (sky) background intensity and a blend parameter which identifies those images that had been deemed by the SuperCOSMOS software to be multiple and subsequently split into component parts. There are no I/SR Survey plates for the outlying field F87, so plates taken for the SERC-I Survey and the AAO-R Survey were used instead. The plates used are listed in Table 2 in order to provide a record of the epoch of the photometry. Finally, data for 14 stars that are too far north for the I/SR Survey were obtained from the on-line SuperCOSMOS Southern Sky Catalogue (which does not include the LMC fields of the I/SR Survey).

A second pair of I/SR plates was available for F56 (R 8215/I8214 taken on 7 Nov. 1982). Measurements of these plates were used to provide a second set of coordinates for comparison purposes. SuperCOSMOS has an internal measuring accuracy of $\leq 0.1$ arcsec and for $\sim 1400$ stars with round images $(\epsilon \geq 0.7)$ the two sets of coordinates matched to $0.15 \pm 0.09$ arcsec. Differences in position of more than 1 arcsec were seen for just $0.25 \%$ of the stars, due to different deblending of complex images. In 22 instances, the carbon star had no image in the primary dataset, due to the star being strongly variable, to high levels of background nebulosity or to a satellite trail. Coordinates for these were provided by the second dataset. However, no data were available in either dataset for 24 other stars, usually those located in severe nebulosity or within the halo of a nearby bright star. In these cases, approximate coordinates were measured using a cursor on the SuperCOSMOS finding chart of the area.

Table 2. Plates used for the photometric measurements

\begin{tabular}{lllll}
\hline$F$ & Plate & Date & Plate & Date \\
\hline 32 & R 4692 & 7 Dec. 1978 & I 4691 & 7 Dec. 1978 \\
33 & R 4701 & 9 Dec. 1978 & I 4697 & 8 Dec. 1978 \\
34 & R 12985 & 14 Feb. 1989 & I 11584 & 9 Oct. 1988 \\
55 & R 7278 & 9 Oct. 1981 & I 7277 & 9 Oct. 1981 \\
56 & R 4609 & 25 Oct. 1978 & I 4610 & 25 Oct. 1978 \\
57 & R 4718 & 19 Dec. 1978 & I 4717 & 19 Dec. 1978 \\
84 & R 4603 & 24 Oct. 1978 & I 4604 & 24 Oct. 1978 \\
85 & R 7382 & 4 Dec. 1981 & I 7383 & 4 Dec. 1981 \\
86 & R 4713 & 18 Dec. 1978 & I 4723 & 20 Dec. 1978 \\
87 & R 16961 & 19 Feb. 1996 & I 13502 & 18 Dec. 1989 \\
\hline
\end{tabular}




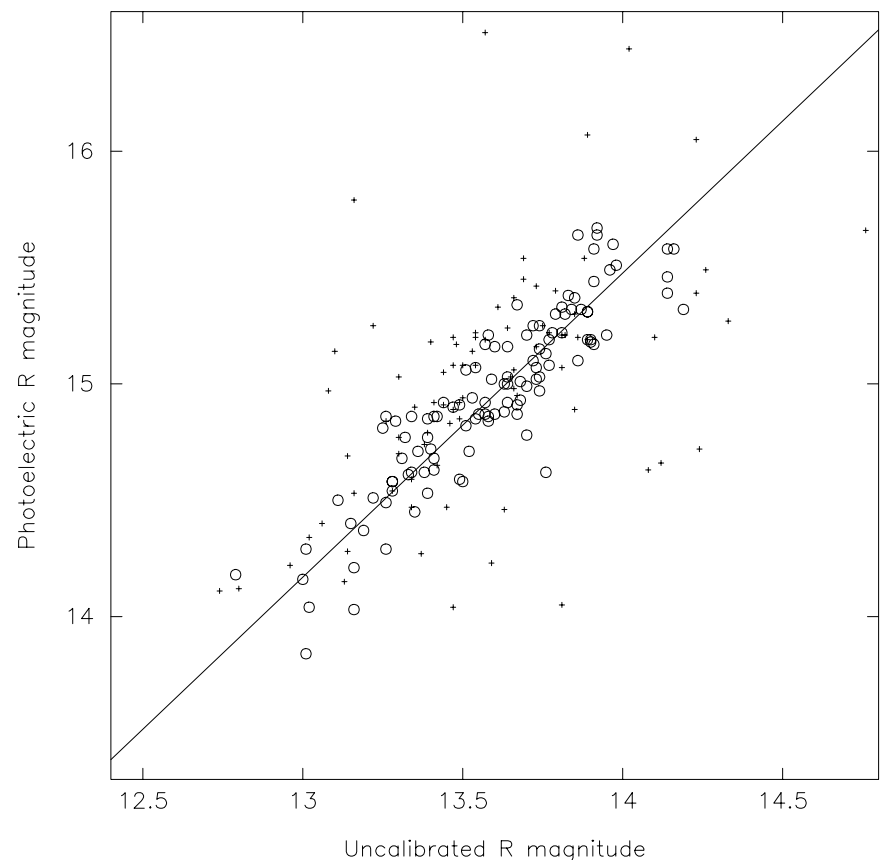

Fig. 1. Calibration of the carbon stars in the $R$ band for F56. The abscissa is the uncalibrated SuperCOSMOS magnitude and the ordinate is the photoelectric measurement by Costa \& Frogel (1996). The small symbols are elliptical and other unused images

\section{Photometric calibration}

The raw $R$ and $I$ SuperCOSMOS measurements of the carbon stars were calibrated using the Kron-Cousins $R I$ photoelectric photometry of carbon stars in the selected regions of Blanco \& McCarthy (1983) as published by Costa \& Frogel (1996). This had to be done in two stages because only the central field (F56) included enough stars to give a reliable calibration. Accordingly, the SuperCOSMOS measurements of F56 were calibrated first and then plate-to-plate photometric comparisons were made in the regions where the other fields overlapped with F56.

Figures 1 and 2 show the least squares calibration fits for F56 in the $R$ waveband and the $(R-I)$ colour respectively. The stars plotted are those with coordinates in the Blanco \& McCarthy (1990) list which match the SuperCOSMOS coordinates to \pm 1 arcsec. The small symbols show stars that were excluded from the fit because of a known photometric problem. They include stars with elliptical images $(\epsilon<0.7)$, and those known to have undeblended companions. Stars seen against gaseous nebulosity or located in vignetted regions of the plates are not shown. The rms scatter about the fitted lines are $0.17 \mathrm{mag}$ in $R$ and 0.14 in $(R-I)$.

Figures 1 and 2 are, in fact, second iterations. It turned out that there was a correlation between $\Delta_{R}$ (the difference between the photoelectric and the calibrated SuperCOSMOS magnitude in $R$ as first derived) and $\Delta_{I}$ (the equivalent quantity in $I$ ), for stars with $\left|\Delta_{R}\right|>$ $0.3 \mathrm{mag}$. This is to be expected for carbon stars and is

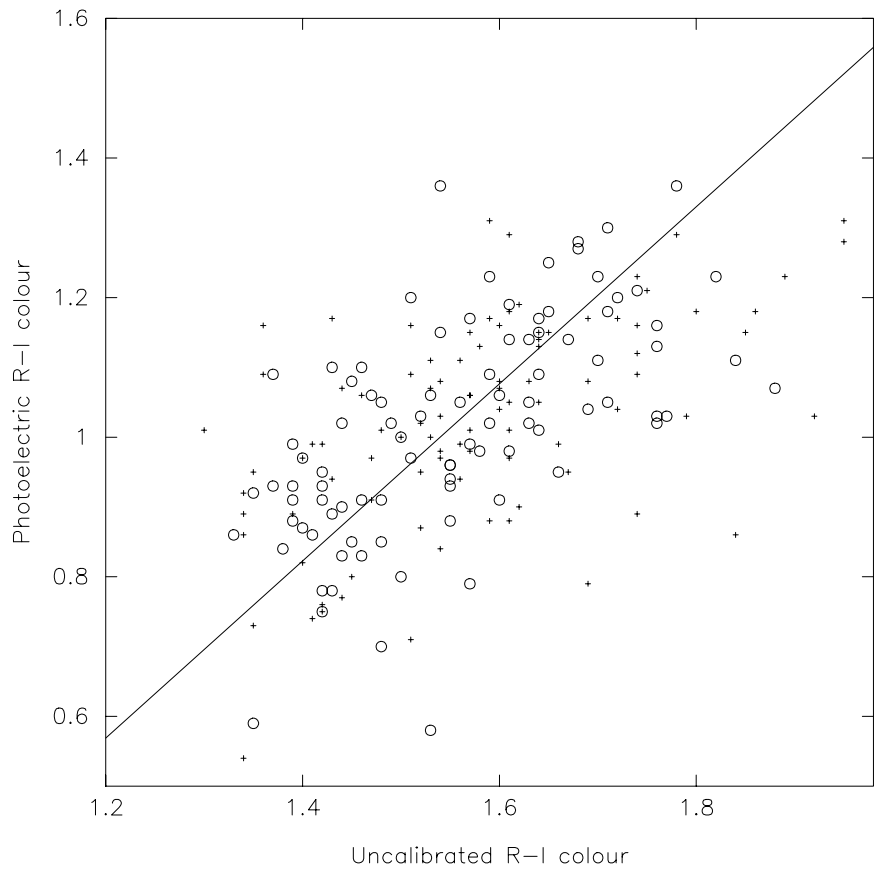

Fig. 2. Calibration of the carbon stars in the $(R-I)$ colour for F56. Details are as for Fig. 1

probably due to the variability of some of them (the $R$ and $I$ plates were taken fairly close in time) and to companion stars affecting in a similar way either the photoelectric photometry or the SuperCOSMOS image deblending procedures. The latter is certainly a real effect because the $\Delta_{R}-\Delta_{I}$ correlation is stronger for elliptical images than for circular ones. Stars with $\Delta_{R} \sim \Delta_{I}$ and $\left|\Delta_{R}\right|>0.3$ were excluded from the final fits. They are some of the stars shown with small symbols in Figs. 1 and 2.

There are small field effects in the data. The deviation from the mean fit for $R$ changes by $\sim 0.1 \mathrm{mag}$ across the plate from centre to edge. Equivalent numbers for $I$ are slightly lower, but in the same sense. This is small and contributes to the residual scatter seen above.

The main UKST fields form a block of nine with F56 central. Consequently, there is a good overlap of F56 with F85 to the north, with F33 to the south, with F57 to the east and with F55 to the west. Calibration of each of these four fields was achieved using all the carbon stars in the appropriate overlap zone. The four corner fields of the block (i.e. those adjacent to the corners of F56 - F32, F34, F84 and F86) were then calibrated twice, using the carbon stars in each of the overlap zones with previously calibrated fields. Figures 3 and 4 show, for $R$ and $I$ respectively, the least squares fits between the SuperCOSMOS uncalibrated magnitudes of those carbon stars that lie in the overlap zone between F85 and F86. The small symbols are for stars with deviant SuperCOSMOS sky parameters due to either excess vignetting or excess nebulosity; they were not used in the fit. The alternative calibrations of the four corner fields differed in $R$ by less than 0.15 mag for input magnitudes between 12 and 14.5 and up to $0.3 \mathrm{mag}$ for the few stars with input magnitudes fainter than this. 


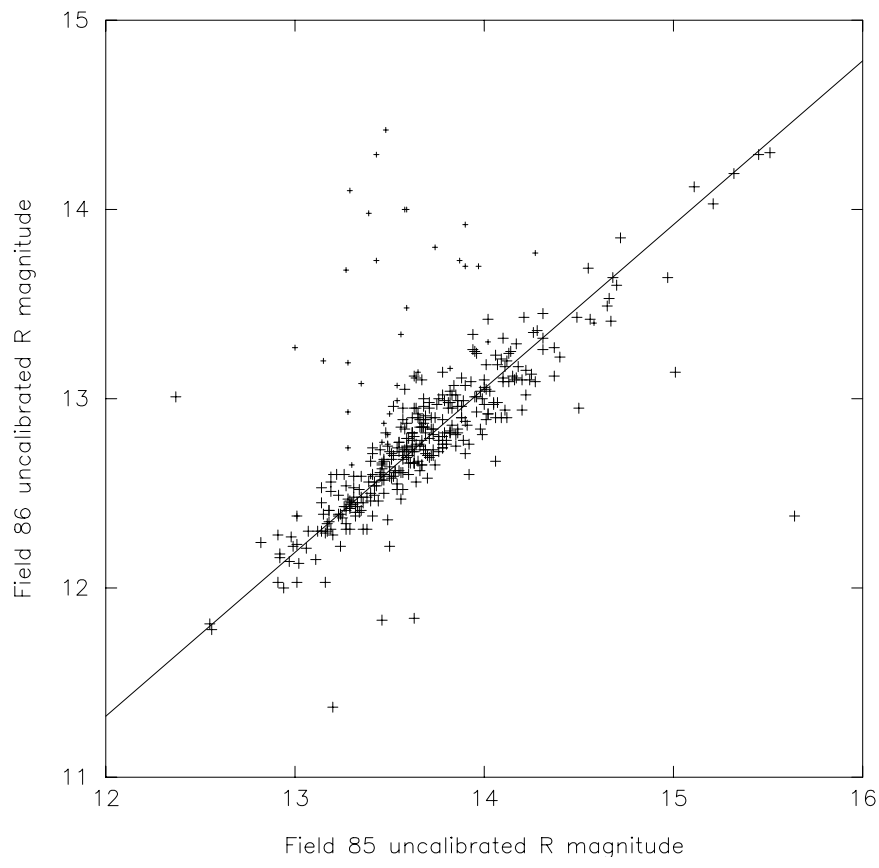

Fig. 3. Uncalibrated $R$-band magnitudes of carbon stars in the overlap zone between F85 and F86. The small symbols are stars affected by vignetting and/or nebulosity

For $I$, these differences were always less than 0.15 mag. With the exception of that between F32 and F55, which has only 6 stars, most overlap zones contain a few hundred stars. So, for F34, F84 and F86, weighted averages of the two alternative calibrations were adopted, the weighting depending on the numbers of stars involved; and for F32, the calibration via F33 was adopted. The tenth UKST field - F87 - was calibrated using its overlap with F86. It is possible that the calibration for this field has the largest systematic errors because it was constructed via three overlap zones and because the direct plate was from a much deeper survey. All stars were then fitted to the F56 standards using these relationships and then converted to the Costa \& Frogel (1996) system using the results of Figs. 1 and 2.

The SuperCOSMOS Southern Sky Catalogue is calibrated and provided the photometry for the 14 northern stars. The $I$-band photometry was from the SERC-I Sky Survey and the $R$-band data from the ESO-R Southern Sky Survey.

As a final check on these procedures, the Costa \& Frogel (1996) standards which are available on these other fields were matched with their calibrated magnitudes. They were found to agree, with a similar spread to that for F56, again with the deviations in $R$ correlating with those in $I$, again presumably due to variability and blending errors.

\section{The Catalogue}

The Catalogue of carbon stars is provided in Table 3 in electronic form. It contains the following informa-

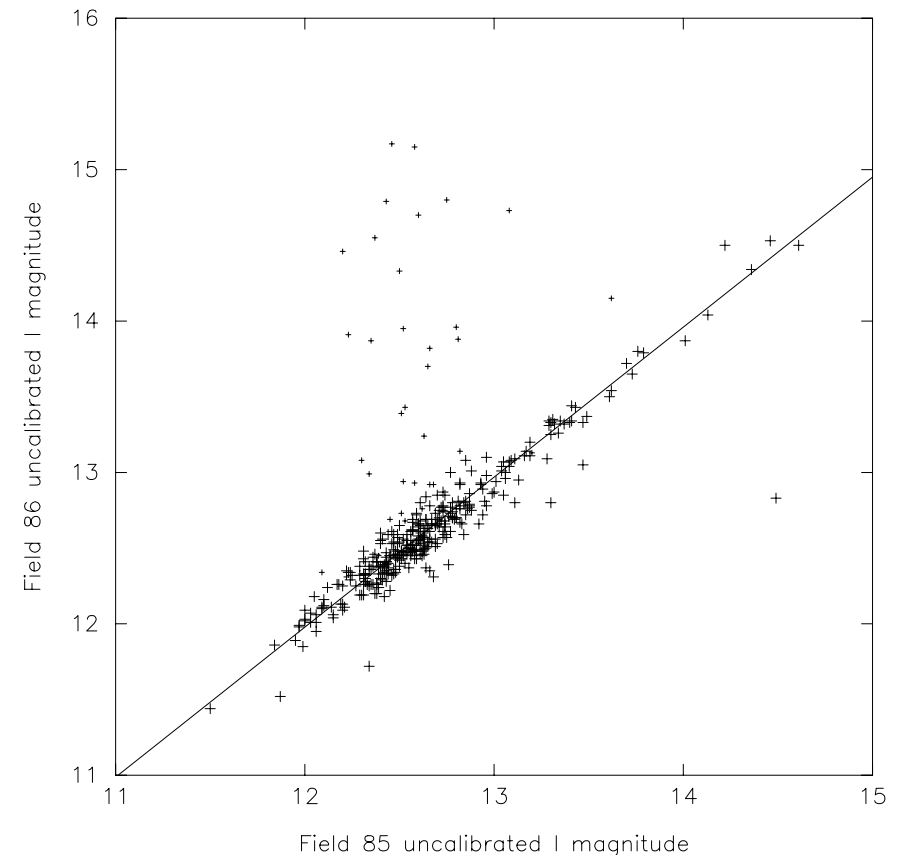

Fig. 4. Uncalibrated $I$-band magnitudes of carbon stars in the overlap zone between F85 and F86. The small symbols are stars affected by vignetting and/or nebulosity

tion: an identification number, J2000 equatorial coordinates usually as measured on the SERC-SR Sky Survey, the calibrated $R$ magnitude and $(R-I)$ colour from the SuperCOSMOS measurements, the field number from which the astrometry and photometry were measured, 5 codes of comments on the data and cross-identifications with Kunkel et al. (1997) and Blanco \& McCarthy (1983). Known symbiotic stars are given cross-identifications from Morgan (1992). "SC" in the field column identifies those stars for which data were extracted from the SuperCOSMOS Southern Sky Catalogue.

Full details of the five codes are given in Table 4 which is available with Table 3. Briefly, there is (1) a photometry code which notes images with companions that are unreliably deblended, (2) an astrometry code which notes images where the coordinates in $R$ and $I$ differ by more than 1 arcsec, (3) a spectrum code which identifies stars for which identification as carbon is less reliable or for which the bands appear weak, (4) a general code to indicate possible contamination by nebulosity or a nearby bright star, galaxy, satellite trail or cluster, and the possibility that the star is variable, and (5) an identification code to indicate stars with companions that are resolved and hence photometrically reliable but might cause confusion for repeat observation. Stars identified by the last code are listed in Table 5 which gives the magnitude difference between the carbon star and its neighbouring and the direction and extent of the separation between the two.

In a few instances it proved impossible to provide photometry, usually due to intense nebulosity or a close bright 
star halo. Such stars have entries of -9.99 for their magnitude or colour.

The existence of an unresolved companion was determined by comparing the pixel image of the OR companion film to the AAO-H $\alpha$ Survey (Parker et al. 1998) as measured by SuperCOSMOS with the appropriate finding chart constructed from the SuperCOSMOS parametric data. These OR films match the SR Survey plates but have a better resolution. Clearly, this procedure will not identify those carbon stars that are binaries or have lineof-sight companions separated by less than 1 arcsec.

\section{Completeness of the survey}

The survey will be incomplete for the following reasons: inconsistent plate quality, overlapping spectra, stellar variability, detection error and selection effects such as limiting magnitude changes due to colour and $C_{2}$-band strength. The cumulative effect of these can be seen by looking at the internal consistency of the identifications and by comparing the results with those of other authors.

\subsection{Internal consistency}

The completeness of the present work can be estimated from the statistics of the detection levels in regions common to neighbouring UKST plates and from repeat scans by independent colleagues. Apart from the very crowded region of the Bar, typical differences in detection level are $\sim 10 \%$ depending on the plate quality and local image crowding. Most of the omissions were due to overlapping spectra; other causes were variability and the difficulty of maintaining a uniform selection criterion for stars with weak Swan bands or faint magnitudes. Most of the stars omitted from one plate as a consequence of being obscured by a neighbouring image were easily seen on the second plate because the direction of the spectral dispersion changes significantly with RA across each plate.

\subsection{Follow-up spectroscopy}

About 1500 of the stars in Table 3 were observed with the $2 \mathrm{dF}$ multi-object spectroscopy system on the AngloAustralian Telescope in January and November 1998 (Cannon et al. 1999) in the wavelength range 5600-6700 $\AA$. Only $\sim 6 \%$ failed to record carbon star spectra. About two thirds of these had extremely weak signals due to fibre misalignment, intrinsic faintness or stellar variability, or spoiling by scattered light. Of the remaining $2 \%$, half could be described as having non-carbon spectra, and even some of these could have arisen from very close companions too close to be deblended by the SuperCOSMOS software. The remaining $1 \%$ were caused by errors in the final identifications which, at the plate scale of the UKST, are difficult in crowded fields.

\subsection{Comparison with Blanco et al.}

The stars of Table 3 were matched with those of Blanco \& McCarthy (1983) - BM during the calibration process. For most BM fields, Table 3 includes $80 \%$ of the BM stars. A poorer match occurred for those BM fields with more than 40 stars where losses due to image overlap are greatest, and for a few very low density fields where low number statistics become significant. A check of two moderate density BM fields showed that the BM stars absent from Table 3 were either absent from the objectiveprism plates or appeared without detectable carbon star features. Conversely, some stars from Table 3 were not seen in the BM data, but statistics are not useful because the precise boundaries of the BM fields are not known. Similarly, a comparison of the present survey with that of Blanco et al. (1980) showed a common population of $\sim 70 \%$ in the crowded "Bar-West" field. This is the level of agreement expected between surveys carried out in different wavebands (Blanco \& McCarthy 1990 - blue-green for the present survey and far-red for the older work).

\subsection{Comparison with the 2-MASS Survey}

Infrared photometry of sources in much of the LMC is now available through the Second Incremental Data Release of the Two Micron All Sky Survey (2-MASS) and is available on-line at http://irsa.ipac. caltech.edu/ (Magellanic Clouds Newsletter No. 42, 2000). JHK data for a small circular area of diameter $2 \mathrm{deg}$, centred on $5.17^{\mathrm{h}},-71.4^{\circ}$ were downloaded from this site and matched with the SuperCOSMOS data using a coordinate search box of \pm 1 arcsec. (The pairing was successful for $98 \%$ of the objects with a mean positional difference of 0.3 arcsec.) The $K$ versus $(J-K)$ colour-magnitude diagram is shown in Fig. 5 with the carbon stars shown as circles. It is clear that, while most of the stars at the bright end of the red arm are carbon stars, there are many fainter carbon stars that lie on the main body of stars. A similar result holds for the $(J-H)$ versus $(H-K)$ colour-colour diagram.

The $J H K$ data also give another indication of the completeness of Table 3 . Of the 236 stars in the selected area with $(J-H)>0.89$ and $(H-K)>0.32$ (i.e. are highly likely to be carbon stars), 42 are not in Table 3 . These 42 stars were then identified on the objective-prism plates with the result that 20 were considered not to be carbon stars, 6 were too faint to be classified, 3 were absent (much fainter than the direct image of the SR survey plate would suggest) and therefore likely to be variable, 9 were not seen due to spectrum overlap and just 4 were perhaps carbon or carbon variable stars. Thus, no more than 22 out of $236(9 \%)$ were missed. The area selected was in a fairly crowded region on the southern edge of the Bar.

In contrast, $17 \%$ of the carbon stars from Table 3 in this area had infrared colours outside these bounds (i.e. $(J-H)<0.89$ or $(H-K)<0.32)$ where distinction from other stars is impossible. 


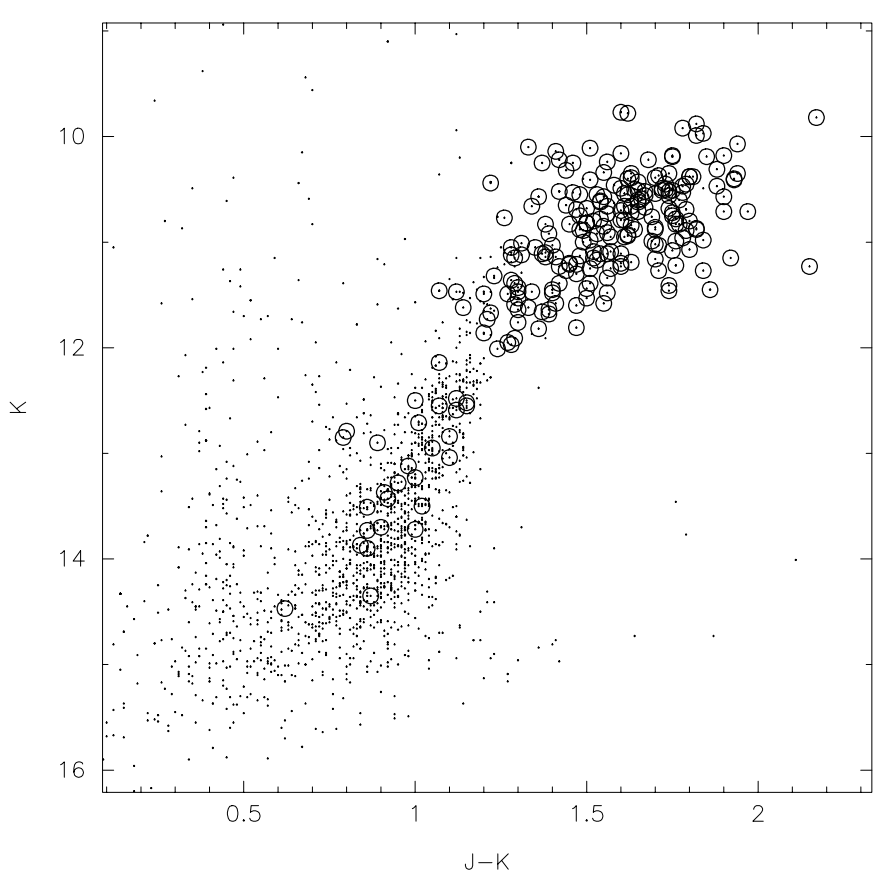

Fig. 5. Infrared colour-magnitude diagram from the 2-MASS Survey for a 2 deg area south of the Bar. The circles are carbon stars from Table 3

\subsection{Comparison with Kunkel et al.}

Kunkel et al. (1997) - KID have presented lists of carbon stars found in the outer regions of both the SMC and the LMC from the photometric selection of very red stars. They also give radial velocities for these stars. It is instructive to compare the results of the two search methods. When this was done for the Inter-Cloud region by Morgan \& Hatzidimitriou (1995) using the photometrically selected sample from Demers et al. (1993), it was found that two thirds of the stars identified on the objective-prism plates were too blue for the photometric selection and one half of the latter were too faint or had bands that were too weak to be identifiable on the objective-prism plates.

The LMC datasets are less well suited for comparison because the Kunkel et al. data are not complete in the innermost regions they covered (the photometric selection was complete but the necessary spectroscopic follow-up was not). The number of obvious carbon stars found on the objective-prism plates is at least twice the number of KID stars in the common areas, but it cannot be assumed that this is because many were missed by KID though being too blue. However, the objective-prism survey is meant to be complete, and the KID stars missing from it can be counted. This was done and the images sought on the objective-prism plates. It turned out that $82 \%$ of the possible 393 KID stars were found. Of the remaining $18 \%$, only $5 \%$ were identifiable as probable carbon stars, and, of those only one quarter were clearly missed (i.e. $1.25 \%$ ), one quarter almost entirely obscured due to over- lapping images and one half were very faint. The other $13 \%$ were considered to be M-type or red stars, or perhaps carbon stars with imperceptible bands, or too faint for the objective-prism plates (i.e. if the red stars were not the correct identifications). It turned out that most of the KID stars considered on the objective-prism plates to be M-type or just red are labelled "Wk C" or "M" by KID and almost all the latter are excluded from Table 3.

In the aforementioned programme of spectroscopy using $2 \mathrm{dF}$ (Cannon et al. 1999), 18 KID objects were observed including four which were not in Table 3 . One of the four was missed during the objective-prism plate searches due to overlap and is a carbon star. One (C0529-7525) is an M-star and is noted in KID as "Wk C", and another (C0526-7529) is very faint on the objective-prism plates and fails to record a useful $2 \mathrm{dF}$ spectrum. The fourth has an extremely weak Swan band at $5635 \AA$ and could not have been included in Table 3 from its objective-prism spectrum. Therefore, better spectra near the Swan bands are needed to confirm these "Wk C" stars as carbon stars before they can be reliably used for velocity studies.

\subsection{Summary}

From these considerations, it would seem that the Catalogue is complete to a level of $\sim 90 \%$ in the uncrowded regions, $\sim 80 \%$ in crowded regions, and perhaps $\sim 70 \%$ in the densest parts of the Bar. These figures, of course, just apply to carbon stars within the selection limits of blue magnitude and $C_{2}$-band strength. Searches carried out in the red or infrared have different selection limits which typically result in gains and losses at the $\sim 10 \%$ level.

\section{Distribution of carbon stars}

Nearly 8000 new carbon stars have been identified in the present programme. The surface distribution of these is shown in Figs. 6 and 7. In Fig. 7 it is as an isodensity contour map constructed from a square surface density grid of element size $40 \mathrm{arcmin}$. This element size was chosen to illustrate the outer parts of the LMC and is not appropriate for the inner parts where the effect of the very crowded and nebulous regions is apparent.

It is clear that carbon stars are found farther than $9^{\circ}$ from the centre of the LMC and not just in the InterCloud region where they are already known to exist (not shown). This has also been seen in the data presented by KID. It is important to point out that the outermost contours resemble the outer star cluster system (Kontizas et al. 1990), though they do have a slightly more face-on orientation. This is consistent with the fact that the LMC clusters fall within an age range comparable to that of the carbon stars. There are other features of note: (1) the region around the Bar has a very high density of carbon stars, especially at its south-eastern end, (2) the density is generally greater in the northern parts than in the areas to the south and south-west, and (3) the centres of the dense parts (the 10 and 20 stars per pixel contours) are 


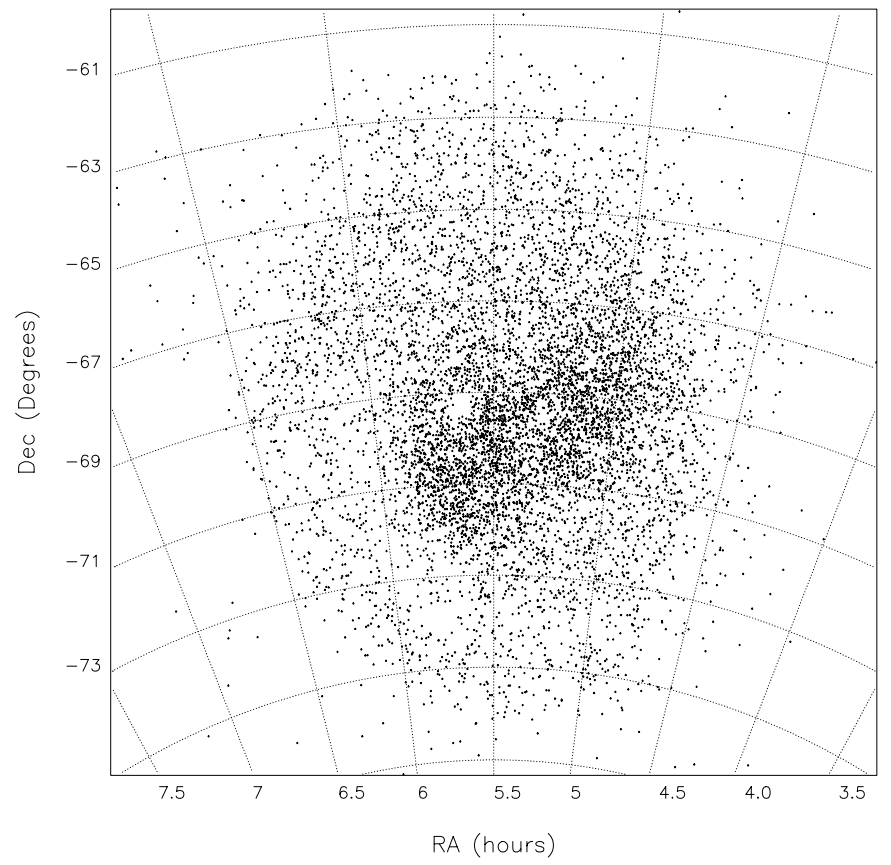

Fig. 6. Distribution of carbon stars in the LMC

found significantly to the east of the centres of the three lower contours, as was found with the clusters (Kontizas et al. 1990) and planetary nebulae (Morgan 1994). More detailed conclusions will be the subject of a further paper.

Acknowledgements. The authors are grateful to the staffs of the UK Schmidt Telescope Unit at the Royal Observatory Edinburgh and the Anglo-Australian Observatory for providing copies of the excellent film material on which the project was based, to the SuperCOSMOS Unit of the ROE for providing the measurements, to the 2-MASS project for the infrared data, and to the British Council (Athens) and Greek Secretariat of Science and Technology for providing funds to support the project. The authors would also like to thank Miss Claire Mollison, Miss Helen Gibson and Dr. Michael Black for help during the construction of the catalogue.

\section{References}

Blanco, V. M., McCarthy, M. F., \& Blanco, B. M. 1980, ApJ, 242,938

Blanco,V. M., \& McCarthy, M. F. 1983, AJ, 88, 1442 - BM

Blanco, V. M., \& McCarthy, M. F. 1990, AJ, 100, 674

Cannon, R. D., Offer, A., Hatzidimitriou, D., Croke, B., \& Morgan, D. H. 1999, in The Stellar Content of Local Group Galaxies, ed. P. Whitelock, \& R. D. Cannon, IAU Symposium, 192, in press

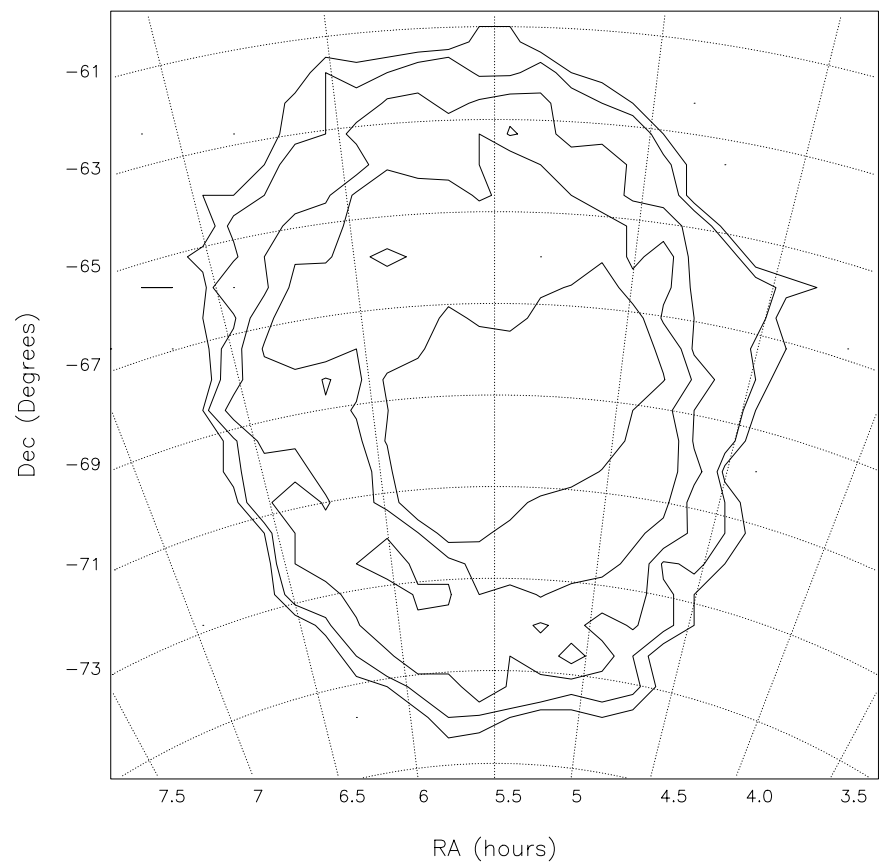

Fig. 7. Isodensity contour map for the carbon stars in the LMC using a pixel element $40 \times 40 \operatorname{arcmin}^{2}$. The contours are 1, 2, 5, 10 and 20 carbon stars per pixel

Costa, E., Frogel, J. A. 1996, AJ, 112, 2607

Dapergolas, A., Morgan, D. H., Kontizas, E., \& Kontizas, M. 1996, in Wide-Field Spectroscopy, ed. E. Kontizas, et al., Astrophys. Space Sci. Lib., 212, 181

Demers, S., Irwin, M. J., \& Kunkel, W. E. 1993, MNRAS, 260, 103

Hatzidimitriou, D., Croke, B. F., Morgan, D. H., \& Cannon, R. D. 1997, A\&AS, 122, 507

Kontizas, M., Morgan, D. H., Hatzidimitriou, D., \& Kontizas, E. 1990, A\&AS, 84, 527

Kunkel, W. E., Irwin, M. J., \& Demers, S. 1997, A\&AS, 122, $129-$ KID

Morgan, D. H. 1992, MNRAS, 258, 639

Morgan, D. H. 1994, A\&AS, 103, 235

Morgan, D. H., \& Hatzidimitriou, D. 1995, A\&AS, 113, 539

Morgan, D. H., Tritton, S. B., Savage, A., Hartley, M., \& Cannon, R. D. 1992, in Digitised Optical Sky Surveys, ed. H. T. MacGillivray, \& E. B. Thomson, Ap\&SS Lib., 174, 11

Parker, Q. A., \& Phillipps, S. 1998, PASA, 15, 28

Rebeirot, E., Azzopardi, M., \& Westerlund, B. E. 1993, A\&AS, 97, 603

Savage, A., Waldron, J. D., Morgan, D. H., et al. 1985a, The UKST Objective Prisms: II. Catalogue of Objects and Technical Data. Royal Observatory Edinburgh

Savage, A., Waldron, J. D., Fretwell, M., et al. 1985b, The UKST Objective Prisms: III. Illustrations of Spectra, Royal Observatory Edinburgh 